\title{
BMJ Open Effectiveness of two psychological interventions for pain management, emotional regulation and promotion of quality of life among adult Portuguese men with haemophilia (PSY- HaEMOPEQ): study protocol for a single-centre prospective randomised controlled trial
}

Patrícia Ribeiro Pinto, ${ }^{1,2}$ Ana Cristina Paredes, ${ }^{1,2}$ Patrício Costa, ${ }^{1,2,9}$ Manuela Carvalho, ${ }^{10}$ Manuela Lopes, ${ }^{10}$ Susana Fernandes, ${ }^{10}$ Susana Pedras, ${ }^{11}$ Armando Almeida ${ }^{1,2}$

To cite: Pinto PR, Paredes AC, Costa $P$, et al. Effectiveness of two psychological interventions for pain management, emotional regulation and promotion of quality of life among adult Portuguese men with haemophilia (PSY-HaEMOPEQ): study protocol for a singlecentre prospective randomised controlled trial. BMJ Open 2017;7:e016973. doi:10.1136/ bmjopen-2017-016973

- Prepublication history for this paper is available online. To view these files please visit the journal online (http://dx.doi. org/10.1136/bmjopen-2017016973).

Received 23 March 2017 Revised 24 May 2017 Accepted 12 June 2017

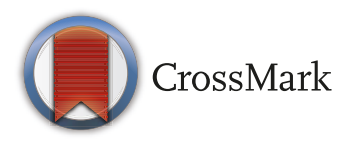

For numbered affiliations see end of article.

Correspondence to Dr Patrícia Ribeiro Pinto; patipinto@med.uminho.pt

\section{ABSTRACT}

Introduction Haemophilia is a bleeding disorder associated with significant pain, emotional distress, quality of life (QoL) impairment and considerable healthcare costs. Psychosocial health and effective pain management are considered essential end points for optimal haemophilia care, but there is a significant gap in evidence-based treatments targeting these outcomes in people with haemophilia (PWH). Psychological interventions are costeffective in promoting emotional well-being, QoL and pain control, although these have been scarcely used in haemophilia field. This investigation aims to evaluate the effectiveness of two psychological interventions for pain management, emotional regulation and promotion of $\mathrm{QoL}$ in $\mathrm{PWH}$.

Methods and analysis This is a single-centre parallel randomised controlled trial conducted at a European Haemophilia Comprehensive Care Centre in Portugal, with five assessment points: baseline (T0), postintervention (T1), 3 (T2), 6 (T3) and 12 (T4) months follow-up. Eligible adult males, with moderate or severe haemophilia A or B will be randomised to experimental (EG) or control (CG) group. Intervention is either cognitive-behavioural therapy (EG1) or hypnosis (EG2), both consisting of four weekly sessions following standardised scripts delivered by trained psychologists. Randomisation will be computer generated, allocation concealment will be guaranteed and outcome assessors will be blind to EG/CG allocation. Main outcomes are pain and haemophilia-related QoL and secondary outcomes include clinical (clotting factor replacement consumption, joint bleeding episodes, analgesic intake) and psychological (pain coping strategies, anxiety, depression, illness perceptions) variables, functional assessment of the joints, inflammatory biomarkers (cytokines, high-sensitivity $C$ reactive protein) and white blood cell count.
Strengths and limitations of this study

- This is an innovative investigation, applying two empirically sound interventions (cognitivebehavioural therapy and hypnosis) to people with haemophilia.

- SPIRIT (Standard Protocol Items Recommendations for Interventional Trials) checklist guidelines were followed, to ensure quality in all aspects of study planning and execution.

- Random allocation and specific efforts to limit bias (blinded outcome assessment, standardisation of intervention and collection of concomitant treatment) contribute to strengthen the study.

- Main limitation of this randomised controlled trial is being a single-centre study, limiting the generalisability of potential findings.

Ethics and dissemination This study was approved by the competent authorities and all procedures will comply with international ethical guidelines for clinical studies involving humans. Written informed consent will be obtained from all participants. The dissemination plan includes peer-reviewed scientific publications, conference participation and web and media coverage. Trial registration number NCT02870452.

\section{BACKGROUND AND RATIONAL}

Haemophilia is an inherited X-linked bleeding disorder caused by a deficiency in coagulation factor VIII (haemophilia A) or IX (haemophilia B). Due to this deficit in 
coagulation factor, the main clinical manifestation of haemophilia is an increased bleeding tendency, either spontaneous or related to trauma or surgery. Spontaneous bleeding episodes occur mainly in the joints (haemarthrosis) and, if recurrent, lead to persistent joint damage and development of chronic joint arthropathy (haemophilic arthropathy). ${ }^{1}$ Severity of haemophilia is classified according to clotting factor level, being defined as mild (clotting factor between $5 \%$ and $40 \%$ of normal), moderate $(1 \%-5 \%$ of normal clotting factor) or severe (clotting factor level under 1\% of normal), which generally correlates to a correspondent increase in bleeding frequency. $^{2}$

Given the clinical presentation of haemophilia, the main goal of care is prevention and treatment of bleeds, which is mainly achieved through different modalities of clotting factor replacement therapy. However, recent guidelines have also highlighted the importance of considering psychosocial health and quality of life (QoL) as important outcomes for optimal care among people with haemophilia $(\mathrm{PWH}) .{ }^{34}$ In fact, $\mathrm{PWH}$ have particular psychological and social needs related to haemophilia-specific threats and challenges, such as pain and daily living restrictions, ${ }^{5}$ which impact significantly on QoL. ${ }^{6}$ Therefore, the focus of current haemophilia management practise is to minimise joint disease and to simultaneously increase QoL. ${ }^{7}$

In this context, a very relevant issue is pain, which is a common, highly debilitating feature of haemophilia that has been related with decreased QoL. ${ }^{8}$ PWH experience acute pain during haemarthrosis, but might also report chronic pain resulting from haemophilic arthropathy. ${ }^{19}$ On a recent 10 country survey, chronic pain related to haemophilia was reported by $38 \%$ of the respondents, highlighting the high prevalence of this condition among PWH. ${ }^{4}$ Nevertheless, patient reports also account for suboptimal pain management, with 33\%-39\% of patients in the USA and Europe reporting dissatisfaction with current pain treatment. ${ }^{40-12}$ This question is such an important issue that, in an editorial, Humphries and Kessler ${ }^{13}$ emphasise that the improvement of pain assessment, prevention and control is a key end point in the development of future treatments for PWH. In sum, pain control should be a priority in haemophilia treatment, ${ }^{13}$ focusing on chronic pain management, and on its prevention, as recommended by international guidelines, which state that non-pharmacological treatments, such as psychological interventions, should be considered for both these purposes. ${ }^{810}$ However, despite well-established recommendations, there is still a scarcity of evidence-based treatment guidelines for haemophilia pain management. This is one important limitation to treatment progress in this field, justifying the need to conduct robust intervention-type investigations in this population. ${ }^{8}$

Another noteworthy issue is that psychological or psychiatric conditions are reported by $47 \%$ of $\mathrm{PWH}$, with $29 \%$ relating these symptoms to haemophilia. ${ }^{4}$ This is even more relevant considering that psychological factors can influence both pain experience and QoL in $\mathrm{PWH} .{ }^{12}$ Interestingly, Cassis et $a t^{6}$ state that variations in QoL are better explained by psychosocial, rather than clinical predictors. Since the former are potentially modifiable through psychological interventions, there is a recognised need to design interventions targeting social and psychological aspects of $\mathrm{PWH} .{ }^{14}$

Indeed, psychological interventions have been proven to be effective in a broad range of disorders and illnesses. ${ }^{15-18}$ Although a few former works have focused on psychological interventions in haemophilia, showing positive and promising results, ${ }^{19-25}$ the lack of recent papers exploring this issue is somewhat surprising, despite the recommendations and guidelines that emphasise their relevance. In those publications, a blend of psychological techniques was applied, with particular emphasis on hypnosis. ${ }^{19} 202425$ In fact, there is considerable evidence for the effectiveness of hypnosis as an empirically supported clinical intervention in managing symptoms such as pain, ${ }^{26-35}$ and also in promoting psychological well-being across a variety of illnesses and disorders. ${ }^{36-43}$ Among PWH, studies have shown that hypnosis can contribute to control pain and to reduce frequency and severity of bleedings and factor consumption. ${ }^{19} 2024$ Concurrently, by promoting better disease management, hypnosis can contribute to better coping and less distress. ${ }^{24}$

Besides hypnosis, cognitive-behavioural therapy is another psychological strategy commonly used in healthcare contexts. This has been the gold standard of psychological intervention, with recognised effectiveness in reducing negative emotions such as anxiety and depression, as well as in managing pain and promoting QoL in chronic disease. ${ }^{15} 1744-48$ Nevertheless, and to the best of our knowledge, it was never fully applied to haemophilia field.

In sum, and despite the shortage of studies focused on psychological interventions in haemophilia, these are recognised as complementary non-pharmacological therapies and as a valuable resource to expand haemophilia care and potentially maximise treatment outcomes, promoting QoL and emotional well-being and improving symptoms management. ${ }^{12} 14$

Another relevant issue in the field of haemophilia concerns inflammatory biomarkers, such as cytokines and high-sensitivity $\mathrm{C}$ reactive protein (hs-CRP), given their recognised role in inflammatory and degenerative processes that are related to the development of haemophilic arthropathy. ${ }^{49}$ For instance, pro-inflammatory (eg, interleukin (IL)-6, IL-1 $\beta$, tumour necrosis factor $(\mathrm{TNF})-\alpha$ ) and anti-inflammatory (eg, IL-10) cytokines have been implicated in the pathophysiology of haemophilic arthropathy, joint pain-associated nociceptive pathways and inhibitor development. ${ }^{49-56}$ In addition, these biomarkers have also been shown to be correlates of psychological variables and, therefore, physiological approaches could support the potential efficacy of psychological interventions on disease and pain control. ${ }^{57-59}$ 
This is particularly relevant in light of the attention being given to psychosocial health in haemophilia, which has been advocated as a priority in the improvement of health status and QoL in PWH. ${ }^{460}$ To this purpose, it is recommended that comprehensive care teams should be multidisciplinary and include a psychosocial expert, who can provide complete assessment of psychosocial status and contribute to an integrated disease management plan. ${ }^{3}$ Globally, integrated care models are preferred over non-integrated care models, but there is still some uncertainty concerning which aspects of care might improve haemophilia management and patient outcomes, and what is the ideal composition of haemophilia care services. ${ }^{61}$ Thus, there is an important gap between the need to clarify these issues and the lack of recent studies analysing psychological interventions for PWH. This, added to the psychosocial impact of haemophilia discussed above, validates the need to advance research in this field, namely through the planning and implementation of clinical randomised controlled trials (RCTs) that test the effectiveness of distinct psychological interventions. In addition, it is noteworthy that, despite pain being recognised as an important consequence of bleeding disorders, it has not been taken into account in most clinical trials of haemophilia. ${ }^{13}$

The current study protocol points to an innovative research that can contribute to better understand the impact and potential benefits of psychological interventions in haemophilia care setting. Given the negative impact of haemophilia on individual QoL and the associated healthcare costs, it is mandatory to evaluate the effectiveness of theoretically grounded psychological interventions in this field.

\section{OBJECTIVE}

The primary objective of this study is to evaluate the relative effectiveness of two psychological interventions, cognitive-behavioural therapy (CBT) and hypnosis (HYP), in order to manage pain, promote emotional regulation and improve QoL, among adult Portuguese men with haemophilia.

\section{Methods}

\section{Trial design}

The design of this study follows the recommendations of Yates $e t a b^{22}$ concerning psychological trials for pain, and reporting of the study results will follow Consolidated Standards of Reporting Trials (CONSORT) guidelines for trials of non-pharmacological interventions. ${ }^{63}$

This is a single-centre three-arm parallel prospective RCT, with one control group (CG) and two experimental groups: CBT and HyP, using an expertise-based RCT design. Participants in both groups will be followed longitudinally, in five time assessment points:

T0: Baseline assessment (preintervention, before randomisation).

T1: Post-test assessment (1 week after intervention).
T2: Follow-up assessment 1 (3 months after intervention).

T3: Follow-up assessment $2 \quad(6$ months after intervention).

T4: Follow-up assessment 3 (12 months after intervention).

\section{Participants and procedures}

According to sample size calculation, 66 patients will enter the study. Estimations were made using $G^{*}$ Power V.3.1.9 and considering the following assumptions: to perform a one-way analysis of variance (ANOVA) with fixed effects, large effect size $(\mathrm{f}=0.4)$, significance level ( $\alpha$-type I error) of 0.05 and statistical power (1- $\beta$-type II error) of 0.80 .

Participants will be recruited at the European Haemophilia Comprehensive Care Centre of São João Hospital Center, in Porto, Portugal. Eligible patients will be identified by the clinicians of the Haemophilia Centre and invited to participate if they comply with the following inclusion criteria: (a) male gender; (b) age $\geq 18$ years; (c) diagnosis of moderate or severe haemophilia A or B, with or without inhibitors; (d) diagnostic of haemophilic arthropathy in at least one joint; (e) chronic pain, as defined by the European Haemophilia Therapy Standardisation Board (EHTSB) ${ }^{10}$; (f) ability to consent voluntary participation to the study; ( $\mathrm{g}$ ) ability to read and write.

The exclusion criteria are: (a) severe and debilitating neurological conditions (eg, dementia); (b) severe psychiatric conditions (eg, schizophrenia); (c) currently undergoing any form of psychotherapy; (d) unavailability to commit to four weekly sessions.

Patients willing to enrol will be screened by the clinicians to assess inclusion criteria and later referred to the investigators, who will describe and explain the study's objectives and interventions and clarify any concern or doubt, emphasising confidentiality and voluntary nature of participation. After acceptance, patients sign the informed consent and baseline assessment is performed (T0). After baseline assessment, participants are randomly assigned to one of the three groups (CBT, HyP or CG) and, for patients in CBT and HyP groups, four weekly individual intervention sessions are scheduled. On the fifth week, all the patients are assessed for post-test assessment (T1). Follow-up assessments will take place at 3 (T2), 6 (T3) and 12 (T4) months after intervention ending for all participants (CBT, HyP and CG). Participant timeline for enrolment, intervention and assessment points is schematised in figure 1 .

\section{Randomisation and allocation}

Randomisation procedures will follow a stratified blocked randomisation process using a computerised random sequence generator. In order to control for potential confounding effects, stratification will be done by haemophilia severity. The generated sequence will be concealed and patient allocation will not be revealed until official enrolment, after consent is given and baseline assessment 


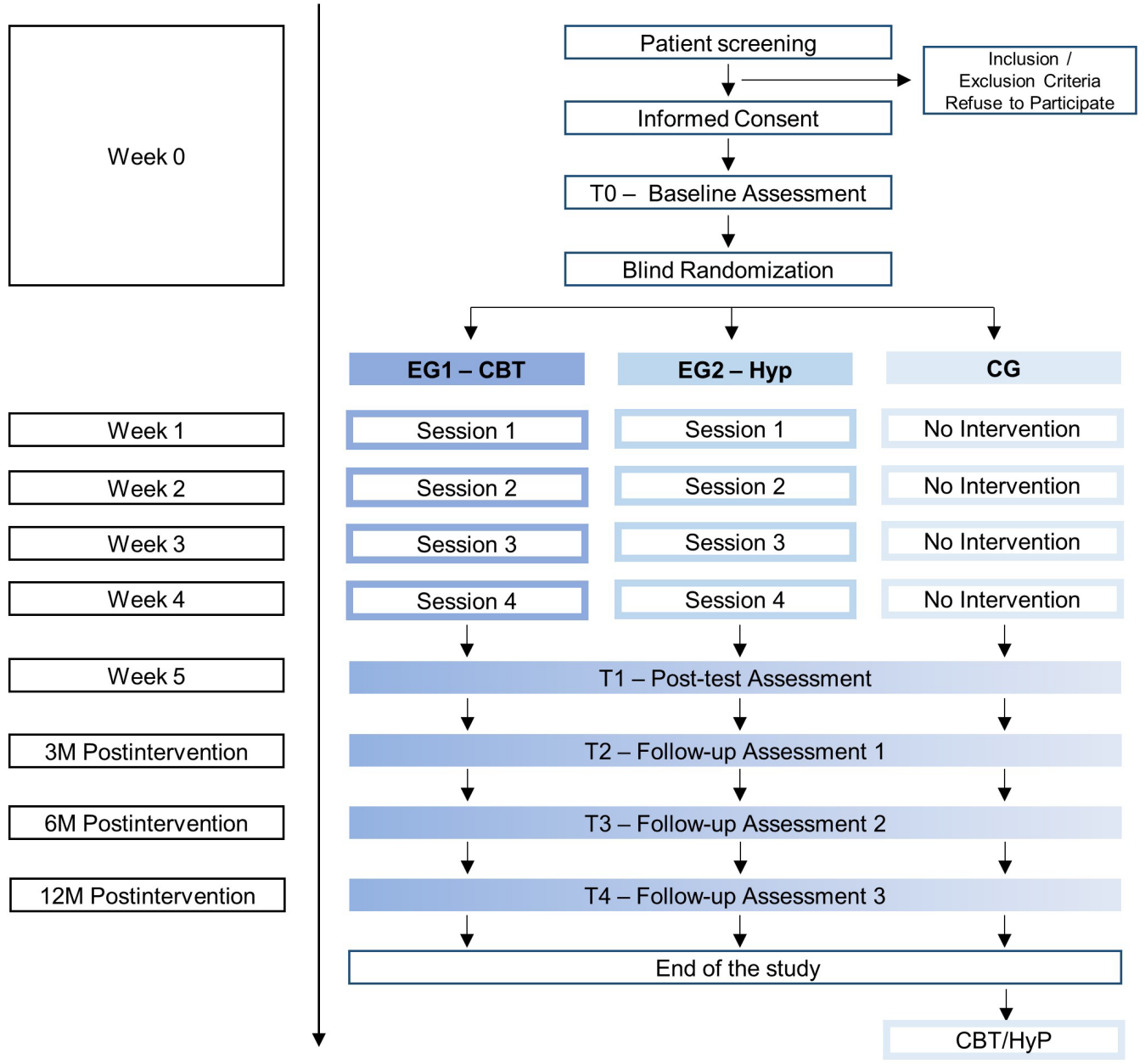

Figure 1 Trial design. CBT, cognitive-behavioural therapy; CG, control group; HyP, hypnosis.

is completed. One of a series of consecutively numbered sealed opaque envelopes with group allocation will be opened at this moment and revealed to the patient. Due to obvious differences in procedure, blinding of the patients to intervention versus control group is impossible. Moreover, it is possible that some patients are familiar with psychological intervention strategies and are able to recognise their allocated intervention and, therefore, blinding to type of psychological intervention (CBT vs HyP) cannot be guaranteed. However, in order to prevent further bias, the type of intervention will not be disclosed to the patients. The different randomisation steps (sequence generation and patient allocation) will be performed independently by the two investigators conducting the intervention sessions, who are aware of patients' allocated arm. Information concerning allocation is concealed from the investigator performing subsequent outcome assessment. There are no anticipated circumstances to justify unblinding of any parties for the duration of the trial, or discontinuation of intervention.

\section{Intervention groups}

The two experimental conditions $(\mathrm{CBT} / \mathrm{HyP})$ have the same format of four consecutive weekly sessions of psychological intervention, scheduled following T0 assessment.
Two doctorate-level health psychologists will conduct these groups individually, in a private and quiet room. Due to the nature of the interventions, each psychologist will perform only one type of intervention $(\mathrm{CBT} / \mathrm{HyP})$, based on training and expertise.

Specific scripts and manuals will be created for each intervention modality, based on theoretical and empirical foundations and taking into account the specificities of haemophilia, its treatment and associated complications.

Strategies to promote participant retention and adherence to intervention and follow-up assessment sessions will include careful explanation of the study and expected benefits, session scheduling according to individual preferences and reminder telephone calls prior to intervention or assessment sessions. In order to control for cointervention bias in the reported outcomes, all concomitant care (clotting factor replacement consumption, analgesics and other medications, medical exams, physical therapy, ice, rest, etc) will be closely monitored, either by self-report or by collecting information from clinical records.

There are no anticipated adverse effects associated with the psychological interventions and/or assessment procedures, but their unlikely occurrence will be carefully monitored. 


\section{Experimental group 1: CBT}

According to this model, thoughts, beliefs, attributions and expectations play a key role in the perception of disease-related symptoms, such as pain, and in how people adjust to them. ${ }^{64}$ The underlying process advocates a strong link between such cognitions and emotional state, physical symptoms and behaviours. Thus, alarming, self-defeating and unrealistic thoughts contribute to negative emotions and behaviour (maladaptive coping responses) while realistic and more reassuring thoughts lead to more positive emotions and behaviour (adaptive coping responses).$^{65} 66$

Furthermore, CBT is a comprehensive approach and enables patients to integrate information on biological, psychological and social influences about disease-related-symptoms, ${ }^{64}$ thereby enhancing their understanding on how the mind and body work together to influence the course of disease and the concomitant pain experience.

This protocol comprises four sessions, one educative and three focused on adaptive coping skills training, wherein active and structured techniques are taught, embracing coping with haemophilia-specific threats, challenges and symptoms flare-ups (eg, bleedings and pain), goal-setting, distraction, relaxation and problem-solving skills.

In this scope, the following contents and strategies will be approached: (1) educational rationale concerning the theoretical assumptions of CBT model, haemophilia characteristics and pain experience (conceptualised as a multidimensional subjective experience, resulting from a dynamic and complex interaction among psychological, biological and social dimensions); (2) cognitive restructuring, with instruction and practise on the identification, challenging and replacing of negative and self-defeating automatic thoughts that may impact on haemophilia symptoms, pain and psychological distress; (3) problem-solving skills, providing patients with an opportunity to deal with the constraints, consequences and implications of haemophilia and (4) relaxation techniques, coupled with attention diversion strategies.

Simultaneously, patients will be encouraged to work toward overall behavioural goals through homework assignments (eg, keep a symptom diary to identify triggers of emotional distress or schedule daily pleasant activities).

\section{Experimental group 2: HyP}

Hypnosis is a psychotherapeutic technique in which the person is guided by the hypnotist to respond to suggestions for alterations in subjective experience, such as changes in sensations, perceptions, emotions, cognitions or behaviours. ${ }^{41}{ }^{67}$ It includes elements such as relaxation, focused attention, imagery, interpersonal processing and suggestion. ${ }^{68}$

Hypnosis interventions usually comprise the following stages: introduction/preparation of the patient (explaining the rationale underlying hypnosis, including dispelling potential myths, misconceptions and doubts); hypnotic induction (suggestions to promote a state of relaxation and focused awareness); imagery (eg, imagining oneself as being in an agreeable and comfortable place); deepening procedure (further suggestions for achieving a more deeply relaxed and focused state); symptom-specific therapeutic suggestions (specific for each illness or disorder, aiming to change or improve symptoms and/or maladaptive behaviours) and conclusion. ${ }^{41}$ Before concluding the process, posthypnotic suggestions might be made, to extend the benefits obtained beyond the session setting. In this line, providing patients with means to perform hypnosis independently by themselves (self-hypnosis) assists in the reinforcement of those posthypnotic suggestions. Indeed, self-hypnosis constitutes a powerful resource that guarantees the practise of the technique, independently and in an autonomous fashion, thereby empowering patients and giving them a sense of control and mastery over their problems and their lives. ${ }^{3065}$

Within this four-session hypnosis intervention, techniques will range from specific direct suggestions for symptom control following hypnotic induction, to a complex sequence of suggestions and metaphors for relaxation, guided imagery, ego strengthening, dissociation and well-being.

In order to engage patients in hypnosis, the first step is to explain its principles, providing patients with a rationale for its learning and use. Moreover, and similarly to what occurs in CBT intervention, the explanation of haemophilia characteristics and pain neurophysiology will be highlighted, emphasising that pain results from a complex and dynamic interplay between biological processes and psychological factors (cognitive and emotional). Symptom-specific suggestions will address haemophilia-specific challenges and threats, treatment-related difficulties, stress-producing situations, bleedings, pain and the emotional reactions to these symptoms, as well as haemophilia adjustment. Specifically concerning pain, the hypnotic suggestions will focus on deep relaxation, sensory substitution, pain intensity reduction, imagined anaesthesia and analgesia (skills for glove analgesia and transfer), decreased pain unpleasantness, managing breakthrough pain and posthypnotic suggestions for effective self-hypnosis. ${ }^{66}$ All suggestions are made on a repetitive basis at each session and all sessions will end with posthypnotic suggestions, underscoring that any experience of well-being, healing and comfort obtained will remain with the patient and last beyond the sessions, becoming a permanent part of how the patient lives life and cope with disease and problematic issues.

To promote the usage and customisation of self-hypnosis, patients will also be given a CD of the session, and encouraged to practise self-hypnosis outside the sessions, at least on a daily basis.

\section{Control group}

Patients in the CG will receive medical treatments and standard care as usual. Assessments will be made in all the same five assessment time points as with EG participants, but without receiving any psychological intervention. At the end of the study, these patients will be given the opportunity to participate in four sessions of the 
intervention that would prove to be most effective at the end of this investigation.

\section{Outcome measures (see table 1) \\ Primary outcome measures}

Pain experience (frequency, intensity and interference) and haemophilia-related QoL, assessed after intervention (T1) and in the follow-up assessments of 3 (T2), 6 (T3) and 12 months (T4) after intervention ending.

\section{Secondary outcome measures}

- Clinical: clotting factor replacement consumption (IU $/ \mathrm{kg} /$ week), joint bleeding episodes and analgesic intake (type, dosage and frequency) assessed at T1, T2, T3 and T4.

- Psychological: anxiety, depression, pain coping strategies and illness perceptions, assessed at T1, T2, T3 and T4.

- Functional: assessment of the joints evaluated at T2 and $\mathrm{T} 4$.

- Physiological: inflammatory biomarkers-cytokines (pro-inflammatory (IL-1 $\beta$, TNF- $\alpha$, IL-6) and anti-inflammatory (IL-10)), hs-CRP and WBC count assessed at $\mathrm{T} 2$ and $\mathrm{T} 4$.

\section{Other variables}

- Sociodemographic (eg, age, professional status) and clinical variables (eg, inhibitor status, prophylaxis) will be taken into account as potential mediators or moderators for the influence of independent variables (type of intervention) on outcome measures.

- Hypnotic susceptibility will be assessed in all patients at baseline (T0) using the Stanford Hypnotic Susceptibility Scale. ${ }^{69}$

\section{Data collection}

All data collection procedures (demographic, clinical, psychological and physiological) will be conducted by trained and experienced healthcare providers. In order to avoid interassessor subjectivity, assessment of the joints will be performed by the same physician, an orthopaedist with experience and training in haemophilia care. To ensure the quality of self-reported data, the psychological assessment will be performed by the same investigator, a trained health psychologist experienced in psychological evaluation procedures. Blood samples will be collected by trained nurses.

\section{Assessment measures}

\section{Sociodemographic information}

- Sociodemographic questionnaire (developed by the research team): collects patients' data concerning age, education, marital status, professional status, household, etc.

\section{Clinical and pain assessment}

- Clinical questionnaire (developed by the research team): gathers general clinical information about patients' haemophilia status, such as type and severity, age at time of diagnosis, type and frequency of medical treatments, clotting factor replacement consumption, inhibitor status, joint bleeding episodes and comorbidities.

- Multidimensional Haemophilia Pain Questionnaire (MHPQ): developed by the research team to assess haemophilia-related pain, following published guidelines for haemophilia pain assessment ${ }^{10}$ and intending to fill a gap in existing pain assessment tools for PWH. ${ }^{13}$ Questionnaire development was based on an extensive literature review, expert opinion, pilot studying and further refinement of item content and wording. It is currently going through the validation process, after being used in its experimental version on the first Portuguese haemophilia national survey conducted by our team.

The MHPQ has 26 items regarding haemophilia-related pain experienced in the previous year. Four items assess the presence of chronic pain according to the EHTSB guidelines, defined as continuous and/or intermittent pain, related to the pathophysiology of haemophilia and requiring pharmacological or non-pharmacological intervention, in which the cause of pain cannot be readily removed, which occurs more than once a week and lasts 3 months or more. ${ }^{10}$ The remaining questions are divided in nine dimensions:

Painful locations: asks about haemophilia-related pain locations, specifying the most painful location and the one which caused the greatest impact.

Duration: assesses how long ago the pain with greatest impact started.

Frequency: evaluates how often the pain is present and when was the last time it occurred.

Triggering factors (and temporal pattern): requires the selection, from a list, of haemophilia pain potential triggers, such as: bleeds, climbing stairs or weather changes, specifying the daytime when pain is most often experienced.

Intensity: measured in regard to specific situations, such as during bleeds, while in rest or during movement, through a 0-10 Numerical Rating Scale (NRS) $(0=$ no pain; $10=$ worst imaginable pain).

Interference: these items were drawn from the Brief Pain Inventory's interference subscale, ${ }^{70}$ evaluating pain interference with general activity, mood, walking ability, normal work, relations with people, sleep and enjoyment of life, assessed according to a NRS ( $0=$ no interference; $10=$ completely interferes).

Strategies for pain control: several strategies are presented (factor replacement, rest, ice, analgesics, distracting, etc) for people to mark the ones they usually do or ever did and the degree of relief they provide $(0 \%-100 \%$ scale).

Pain management specialists: asks about pain specialists people have or would like to consult to help manage pain (eg, haemophilia doctors, anaesthesiologists, psychologists, professionals of alternative therapies, etc).

Satisfaction with pain treatment: evaluates global satisfaction with pain treatment through a single question, on a 


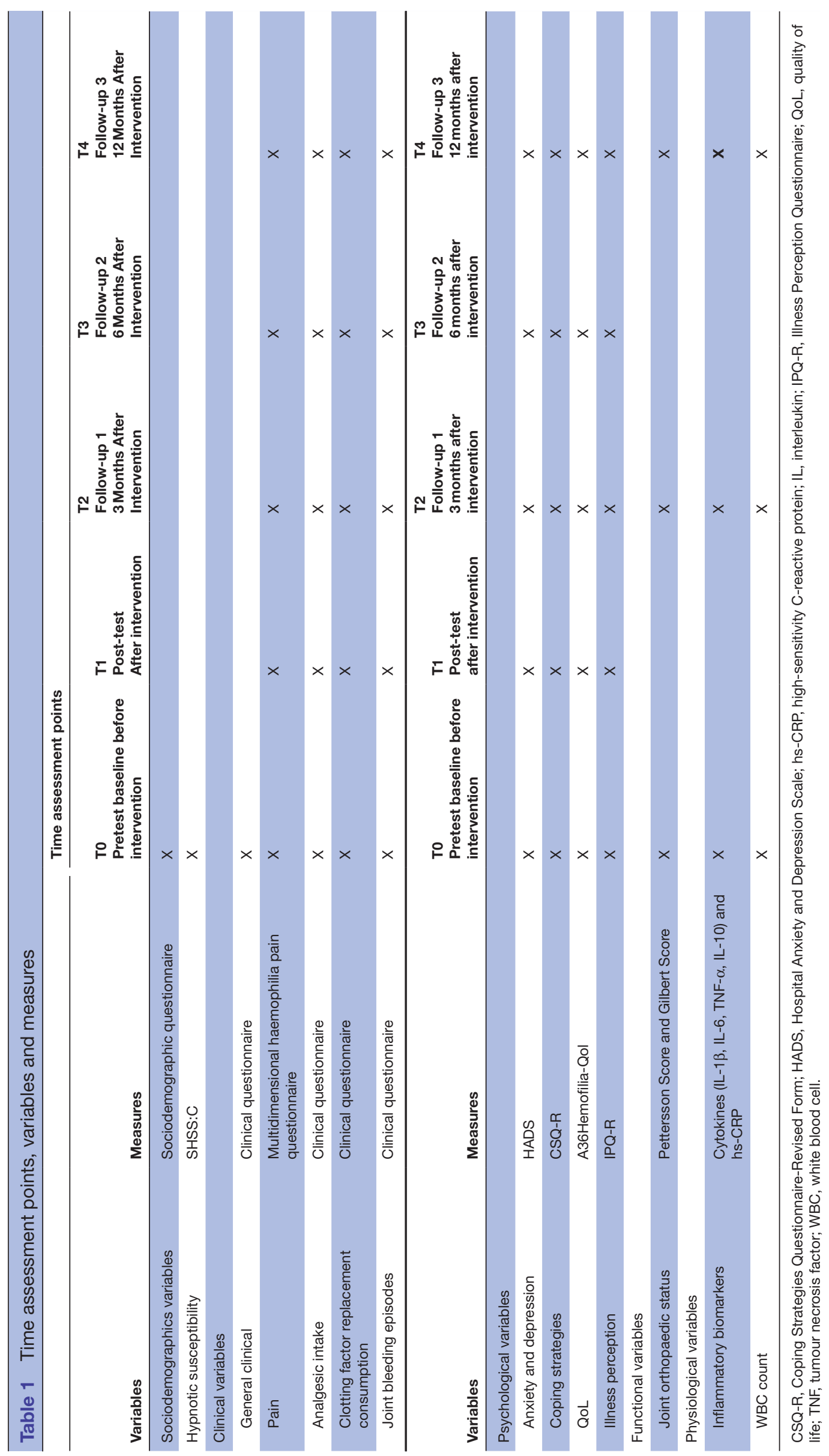


5-point scale (ranging from $1=$ "very dissatisfied' to $5=$ "very satisfied').

Each dimension in analysed separately and no global pain score is computed for the MHPQ.

\section{Psychological assessment}

The Portuguese versions of the following questionnaires will be used.

- A36Hemofilia-Qol ${ }^{71}$ : this is an haemophilia-specific self-report questionnaire assessing health-related QoL. The 36 items are divided in 9 subscales: physical health; daily activities; joints; pain; treatment satisfaction; treatment difficulties; emotional functioning; mental health and relationships and social activity. A total score can also be computed. The A36Hemofilia-Qol was originally developed and validated in Spain with good validity and reliability properties. ${ }^{71}$ The Portuguese version was created following a complete translation back-translation process by certified translators. Similar to the above-mentioned MHPQ, it is currently going through the validation process.

- Hospital Anxiety and Depression Scale ${ }^{72}$ : assesses anxiety and depression in two separate subscales with seven items each. Scoring in each item ranges from 0 to 3 , with a total possible score varying from 0 to 21 . Higher scores translate higher levels of anxiety and depression. This questionnaire was developed in a hospital outpatient clinic, avoiding questions that could be influenced by physical illness symptoms ${ }^{72}$ and has since been found a reliable measure of anxiety and depression symptom severity in physical and psychiatric illness, primary care patients and general population. ${ }^{73}$ It has been validated for Portuguese patients. ${ }^{74}$

- Coping Strategies Questionnaire-Revised Form (CSQ-R $)^{75}$ : includes 27 items that represent different coping strategies people usually use when in pain. It is organised in six subscales: distraction/diverting attention; praying and hoping; ignoring pain sensations; reinterpreting pain sensations; pain-coping self-statements and pain catastrophising. The Portuguese version applied in this study has been used in several investigations in hospital setting with good reliability properties. ${ }^{76-78}$

- Illness Perception Questionnaire (IPQ-R) ${ }^{79}$ : assesses patients beliefs about their illness, according to seven dimensions: timeline acute/chronic; timeline cyclical; consequences; personal control; treatment control; illness coherence; emotional representation. The IPQ-R has been validated for Portugal ${ }^{80}$ and, in this study, participants will be evaluated with a psychometrically shortened version of 21 items, ${ }^{81}$ previously used in Portuguese clinical setting, ${ }^{76-78}$ to reduce respondent burden.

\section{Physiological assessment}

This will be performed through the collection of blood samples in order to conduct WBC count and to achieve a systemic evaluation of pro-inflammatory (IL-6, IL-1 $\beta$,
TNF- $\alpha$ ) and anti-inflammatory cytokines (IL-10), as well as of hs-CRP.

On arrival at the Haemophilia Centre (between 09:30 and 1:30 hours), patients will undergo sample blood collection and EDTA samples will be transported immediately to the lab. In the lab, blood samples are centrifuged $15 \mathrm{~min}$ at $3000 \mathrm{rpm}$, and plasma aliquoted and stored in a freezer at $-70^{\circ} \mathrm{C}$, until further analysis. Plasma levels of cytokines (IL-6, IL-1 $\beta$, TNF- $\alpha$, IL-10) are assayed in duplicate using ultrasensitive multiplex human ELISA kits (Life Technologies).

\section{Functional assessment}

- WFH Physical Examination Score (Gilbert Score ${ }^{82}$ : rates joint impairment based on clinical evaluation of joints, considering physical status evaluation and reported pain. Physical examination includes assessment of swelling, muscle atrophy, axial deformity, crepitus on motion, range of motion, flexion contracture and instability.

- Pettersson Score ${ }^{83}$ : assesses joints quantitatively, based on the presence or absence of radiographic changes in eight dimensions: osteoporosis, enlargement of epiphysis, irregularity of subchondral surface, narrowing of joint space, subchondral cysts formation, erosion of joint margins, gross incongruence of articulating bone ends and joint deformity (angulation and/or displacement between articulating bones).

\section{Data analysis plan}

All data analysis procedures will be performed using IBM SPSS Statistics V.24 (SPSS, Chicago, Illinois, USA), except for sample size estimation, that will be calculated with G*Power V.3.1.9, as described above.

The analysis plan will follow intention-to-treat principles (all participants as randomised). Frequencies and descriptive statistics (means, SD, skewness and kurtosis) will be analysed for sample characteristics at baseline and for outcome measures in the five assessment points. A mixed ANOVA will be performed to test mean differences between the three groups (CBT vs HyP vs CG; between-subject factor) over the five measurement points (within-subjects factor). This procedure allows the test of main group/intervention and time effects and mainly if there is a significant interaction effect between the two factors (between and the within subjects).

At the end of the study, it will be possible to determine if changes in outcomes (eg, QoL) over time depend on the intervention. If no significant interaction effects are obtained, it can be concluded that changes in outcomes were simply due to time. Effect size measures (partial eta squared) and statistical power (1- $\beta$ ) will be presented for all statistical tests performed. Results will be considered significant for $\mathrm{p}$ values $<0.05$.

Since all data collection procedures will be conducted in-person, there is no anticipated missing data for baseline or subsequent assessments. In the case of missing 
values existence, missing value analysis will be performed to determine if missing observations are: a) completely at random (MCAR) or b) at random (MAR). Missing values replacement will be performed accordingly (MCAR or MAR) using multiple imputation ${ }^{84}$ performed using the IBM SPSS Amos V.24.

\section{ETHICS AND DISSEMINATION}

This investigation will submit to international ethical principles and guidelines for clinical studies involving humans. All participants will read and sign the informed consent and all doubts and questions will be addressed by the research team. Study-related data will be stored in locked cabinets and limited access, password protected computers and confidentiality will be guaranteed by assigning a code to each participant. An anonymised final version of the dataset will be available to team members.

The study was authorised by the Portuguese National Data Protection Agency (CNPD) and approved by the Life Sciences and Health Ethics Subcommittee-University of Minho, and by the Centro Hospitalar de São João-E.P.E. Ethics Committee. Any modification to the research protocol will be communicated in the clinicaltrials.gov RCT registry. Final conclusions of this investigation will be published in peer-reviewed journals and presented at haemophilia international conferences, and made available to the PWH community through appropriate channels (national news channels, web and social media).

\section{Author affiliations}

${ }^{1}$ Life and Health Sciences Research Institute, School of Medicine, University of Minho, Braga, Portugal

2ICVS / 3B's, PT Government Associate Laboratory, Braga / Guimarães, Portugal ${ }^{9}$ Faculty of Psychology and Education Sciences, University of Porto, Porto, Portugal ${ }^{10}$ Centre of Hemophilia, Department of Transfusion Medicine and Blood Bank, Centro Hospitalar São João, Porto, Portugal

${ }^{11}$ School of Psychology, University of Minho, Braga, Portugal

Contributors PRP conceived the study idea and together with SP developed the design and created the intervention manuals. ACP and AA substantially contributed for the development and refinement of the study protocol. MC, ML and SF contributed for the refinement of the design with clinical expertise. PC supervised the power analyses and planned the data analysis. All authors participated in the drafting of this paper and critically revised and approved the final version. All authors are accountable for any issue regarding the accuracy and integrity of the work.

Funding This work is supported by a grant from the Novo Nordisk HERO Research Grant 2015. PRP has a grant (SFRH/BPD/103529/2014) from the Portuguese Foundation of Science and Technology.

Competing interests None declared.

Patient consent Detail has been removed from this case description/these case descriptions to ensure anonymity. The editors and reviewers have seen the detailed information available and are satisfied that the information backs up the case the authors are making.

Ethics approval Life Sciences and Health Ethics Subcommittee, University of Minho; Health Ethics Committee Centro Hospitalar São João.

Provenance and peer review Not commissioned; externally peer reviewed. Open Access This is an Open Access article distributed in accordance with the Creative Commons Attribution Non Commercial (CC BY-NC 4.0) license, which permits others to distribute, remix, adapt, build upon this work non-commercially, and license their derivative works on different terms, provided the original work is properly cited and the use is non-commercial. See: http://creativecommons.org/ licenses/by-nc/4.0/

C Article author(s) (or their employer(s) unless otherwise stated in the text of the article) 2017. All rights reserved. No commercial use is permitted unless otherwise expressly granted.

\section{REFERENCES}

1. Lobet S, Hermans $C$, Lambert C. Optimal management of hemophilic arthropathy and hematomas. J Blood Med 2014;5:207-18.

2. Blanchette VS, Key NS, Ljung LR, et al. Definitions in hemophilia: communication from the SSC of the ISTH. J Thromb Haemost 2014;12:1935-9.

3. Srivastava A, Brewer AK, Mauser-Bunschoten EP, et al. Guidelines for the management of hemophilia. Haemophilia 2013;19:e1-e47.

4. Forsyth AL, Gregory M, Nugent D, et al. Haemophilia experiences, results and Opportunities (HERO) Study: survey methodology and population demographics. Haemophilia 2014;20:44-51.

5. De Moerloose P, Fischer K, Lambert T, et al. Recommendations for assessment, monitoring and follow-up of patients with haemophilia. Haemophilia 2012;18:319-25.

6. Cassis FR, Querol F, Forsyth A, et al. Psychosocial aspects of haemophilia: a systematic review of methodologies and findings. Haemophilia 2012;18:e101-e114.

7. Raffini L, Manno C. Modern management of haemophilic arthropathy. Br J Haematol 2007;136:777-87.

8. Riley RR, Witkop M, Hellman E, et al. Assessment and management of pain in haemophilia patients. Haemophilia 2011;17:839-45.

9. Merskey HE. Classification of chronic pain: descriptions of chronic pain syndromes and definitions of pain terms. Pain, 1986. http:// www.iasp-pain.org/files/Content/ContentFolders/Publications2/ FreeBooks/Classification-of-Chronic-Pain.pdf.

10. Holstein K, Klamroth R, Richards M, et al. Pain management in patients with haemophilia: a European survey. Haemophilia 2012;18:743-52.

11. Witkop M, Lambing A, Divine G, et al. A national study of pain in the bleeding disorders community: a description of haemophilia pain. Haemophilia 2012;18:e115-e119.

12. Young G, Tachdjian R, Baumann K, et al. Comprehensive management of chronic pain in haemophilia. Haemophilia 2014;20:e113-e120.

13. Humphries TJ, Kessler CM. Pain in haemophilia: are we listening? Haemophilia 2016;22:175-8.

14. Cassis F, Emiliani F, Pasi J, et al. Psychological interventions for people with hemophilia. Cochrane Database Syst Rev 2012;11:CD010215.

15. Carlson LE, Bultz BD. Efficacy and medical cost offset of psychosocial interventions in cancer care: making the case for economic analyses. Psychooncology 2004;13:837-49.

16. Crane DR, Christenson JD. The Medical Offset effect: patterns in Outpatient Services Reduction for High Utilizers of Health Care. Contemp Fam Ther 2008;30:127-38.

17. Williams AC, Eccleston C, Morley S. Psychological therapies for the management of chronic pain (excluding headache) in adults. Cochrane Database Syst Rev 2012;11:CD007407.

18. Frederiksen $Y$, Farver-Vestergaard I, Skovgård NG, et al. Efficacy of psychosocial interventions for psychological and pregnancy outcomes in infertile women and men: a systematic review and meta-analysis. BMJ Open 2015;5:e006592.

19. LaBaw WL. Auto-hypnosis in haemophilia. Haematologia 1975;9(12):103-10.

20. Lucas ON. The use of hypnosis in Hemofilia dental care. Ann N Y Acad Sci 1975;20:263-6.

21. Varni JW. Behavioral medicine in hemophilia arthritic pain management: two case studies. Arch Phys Med Rehabil $1981 ; 62: 183-7$

22. Varni JW. Self-regulation techniques in the management of chronic arthritic pain in hemophilia. Behav Ther 1981;12:185-94.

23. Varni JW, Gilbert A. Self-regulation of chronic arthritic pain and longterm analgesic dependence in a haemophiliac. Rheumatol Rehabil 1982;21:171-4.

24. Swirsky-Sacchetti T, Margolis CG. The effects of a comprehensive self-hypnosis training program on the use of factor VIII in severe hemophilia. Int J Clin Exp Hypn 1986;34:71-83.

25. LaBaw W. The use of hypnosis with hemophilia. Psychiatr Med 1992;10:89-98. 
26. Montgomery GH, DuHamel KN, Redd WH. A meta-analysis of hypnotically induced analgesia: how effective is hypnosis? Int J Clin Exp Hypn 2000;48:138-53.

27. Montgomery GH, David D, Winkel G, et al. The effectiveness of adjunctive hypnosis with surgical patients: a meta-analysis. Anesth Analg 2002;94:1639-45.

28. Lang EV, Rosen MP. Cost analysis of adjunct hypnosis with sedation during outpatient interventional radiologic procedures. Radiology 2002;222:375-82.

29. Patterson DR, Jensen MP. Hypnosis and clinical pain. Psychol Bull 2003;129:495-521.

30. Jensen M, Patterson DR. Hypnotic treatment of chronic pain. $J$ Behav Med 2006;29:95-124.

31. Elkins G, Jensen MP, Patterson DR. Hypnotherapy for the management of chronic pain. Int J Clin Exp Hypn 2007;55:275-87.

32. Stoelb BL, Molton IR, Jensen MP, et al. The efficacy of hypnotic analgesia in adults: a review of the literature. Contemp Hypn 2009;26:24-39.

33. Tefikow S, Barth J, Maichrowitz S, et al. Efficacy of hypnosis in adults undergoing surgery or medical procedures: a meta-analysis of randomized controlled trials. Clin Psychol Rev 2013;33:623-36.

34. Adachi T, Fujino H, Nakae A, et al. A meta-analysis of hypnosis for chronic pain problems: a comparison between hypnosis, standard care, and other psychological interventions. Int J Clin Exp Hypn 2014;62:1-28.

35. Kendrick C, Sliwinski J, Yu Y, et al. Hypnosis for acute procedural pain: a critical review. Int J Clin Exp Hypn 2016;64:75-115.

36. Palsson OS, Turner MJ, Johnson DA, et al. Hypnosis treatment for severe irritable bowel syndrome: investigation of mechanism and effects on symptoms. Dig Dis Sci 2002;47:2605-14.

37. Stewart JH. Hypnosis in contemporary medicine. Mayo Clin Proc 2005;80:511-24.

38. Montgomery GH, Bovbjerg DH, Schnur JB, et al. A randomized clinical trial of a brief hypnosis intervention to control side effects in breast surgery patients. J Natl Cancer Inst 2007;99:1304-12.

39. Xu Y, Cardeña E. Hypnosis as an adjunct therapy in the management of diabetes. Int J Clin Exp Hypn 2008:56:63-72.

40. Golden WL. Cognitive hypnotherapy for anxiety disorders. Am J Clin Hypn 2012;54:263-74.

41. Montgomery GH, Schnur JB, Kravits K. Hypnosis for cancer care: over 200 years young. CA Cancer J Clin 2013;63:31-44.

42. Cheseaux N, de Saint Lager AJ, Walder B. Hypnosis before diagnostic or therapeutic medical procedures: a systematic review. Int J Clin Exp Hypn 2014;62:399-424.

43. Corman I, Bouchema Y, Miquel B, et al. HypnosIS to faciLitate transesophageal echocardiograPhy tolerance: the I-SLEPT study. Arch Cardiovasc Dis 2016;109:171-7.

44. White CA. Cognitive behavioral principles in managing chronic disease. West J Med 2001;175:338-42.

45. Halford J, Brown T. Cognitive-behavioural therapy as an adjunctive treatment in chronic physical illness. Advances in Psychiatric Treatment 2009;15:306-17.

46. Morley S. Efficacy and effectiveness of cognitive behaviour therapy for chronic pain: progress and some challenges. Pain 2011;152:S99-S106

47. Hofmann SG, Asnaani A, Vonk IJ, et al. The efficacy of cognitive behavioral therapy: a review of Meta-analyses. Cognit Ther Res 2012;36:427-40.

48. Ehde DM, Dillworth TM, Turner JA. Cognitive-behavioral therapy for individuals with chronic pain: efficacy, innovations, and directions for research. Am Psychol 2014:69:153-66.

49. Jansen NW, Roosendaal G, Hooiveld MJ, et al. Theobald M, Lafeber FP. interleukin-10 protects against blood-induced joint damage. $\mathrm{Br} J$ Haematol 2008;142:953-61.

50. Kapoor M, Martel-Pelletier J, Lajeunesse D, et al. Role of proinflammatory cytokines in the pathophysiology of osteoarthritis. Nat Rev Rheumatol 2011;7:33-42.

51. Valentino LA. Blood-induced joint disease: the pathophysiology of hemophilic arthropathy. J Thromb Haemost 2010;8:1895-902.

52. Acharya SS. Exploration of the pathogenesis of haemophilic joint arthropathy: understanding implications for optimal clinical management. Br J Haematol 2012;156:13-23.

53. Lee AS, Ellman MB, Yan D, et al. A current review of molecular mechanisms regarding osteoarthritis and pain. Gene 2013:527:440-7.

54. Oliveira CA, Velloso-Rodrigues C, Machado FC, et al. Cytokine profile and FVIII inhibitors development in haemophilia A. Haemophilia 2013;19:e139-e142.

55. Miller RE, Miller RJ, Malfait AM. Osteoarthritis joint pain: the cytokine connection. Cytokine 2014;70:185-93.
56. Pulles AE, Mastbergen SC, Schutgens RE, et al. Pathophysiology of hemophilic arthropathy and potential targets for therapy. Pharmacol Res 2017;115:192-9.

57. Miller GE, Cohen S, Ritchey AK. Chronic psychological stress and the regulation of pro-inflammatory cytokines: a glucocorticoidresistance model. Health Psychol 2002;21:531-41.

58. Campbell CM, Edwards RR. Mind-body interactions in pain: the neurophysiology of anxious and catastrophic pain-related thoughts. Transl Res 2009;153:97-101.

59. Antoni MH, Lutgendorf SK, Blomberg B, et al. Cognitive-behavioral stress management reverses anxiety-related leukocyte transcriptional dynamics. Biol Psychiatry 2012;71:366-72.

60. Cassis FR, Buzzi A, Forsyth A, et al. Haemophilia Experiences, Results and Opportunities (HERO) Study: influence of haemophilia on interpersonal relationships as reported by adults with haemophilia and parents of children with haemophilia. Haemophilia 2014;20:e287-e295.

61. Pai M, Key NS, Skinner M, et al. NHF-McMaster guideline on Care models for Haemophilia Management. Haemophilia 2016;22(S3):6-16.

62. Yates SL, Morley S, Eccleston C, et al. A scale for rating the quality of psychological trials for pain. Pain 2005;117:314-25.

63. Boutron I, Moher D, Altman DG, et al. Extending the CONSORT statement to randomized trials of nonpharmacologic treatment: explanation and elaboration. Ann Intern Med 2008;148:295-309.

64. Keefe FJ, Abernethy AP, C Campbell L. Psychological approaches to understanding and treating disease-related pain. Annu Rev Psychol 2005:56:601-30.

65. Jensen MP. A neuropsychological model of pain: research and clinical implications. J Pain 2010;11:2-12.

66. Jensen MP. Psychosocial approaches to pain management: an organizational framework. Pain 2011;152:717-25.

67. Green JP, Barabasz AF, Barrett D, et al. Forging ahead: the 2003 APA Division 30 definition of hypnosis. Int J Clin Exp Hypn 2005;53:259-64

68. Jensen MP, Patterson DR. Hypnotic approaches for chronic pain management: clinical implications of recent research findings. Am Psychol 2014;69:167-77.

69. Weitzenhoffer AM, Hilgard ER. Stanford hypnotic susceptibility scale, form C (Vol. 27). Palo Alto, CA: Consulting Psychologists Press 1962

70. Cleeland CS, Ryan KM. Pain assessment: global use of the brief pain inventory. Ann Acad Med Singapore 1994;23:129-38.

71. Remor E, Arranz P, Quintana M, et al. Psychometric field study of the new haemophilia quality of life questionnaire for adults: the 'Hemofilia-QoL'. Haemophilia 2005;11:603-10.

72. Zigmond AS, Snaith RP. The hospital anxiety and depression scale. Acta Psychiatr Scand 1983;67:361-70.

73. Bjelland I, Dahl AA, Haug TT, et al. The validity of the Hospital anxiety and depression Scale. an updated literature review. J Psychosom Res 2002;52:69-77.

74. Pais-Ribeiro J, Silva I, Ferreira T, et al. Validation study of a Portuguese version of the Hospital anxiety and depression Scale. Psychol Health Med 2007;12:225-37.

75. Riley JL, Robinson ME. CSQ: five factors or fiction? Clin J Pain 1997:13:156-62.

76. Pinto PR, McIntyre T, Almeida A, et al. The mediating role of pain catastrophizing in the relationship between presurgical anxiety and acute postsurgical pain after hysterectomy. Pain 2012;153:218-26.

77. Pinto PR, Mclntyre T, Araújo-Soares V, et al. Differential predictors of acute post-surgical pain intensity after abdominal hysterectomy and Major joint arthroplasty. Ann Behav Med 2015;49:384-97.

78. Pinto PR, Mclntyre T, Nogueira-Silva C, et al. Risk factors for persistent postsurgical pain in women undergoing hysterectomy due to benign causes: a prospective predictive study. J Pain 2012;13:1045-57.

79. Moss-Morris R, Weinman J, Petrie K, et al. The revised illness perception questionnaire (IPQ-R). Psychol Health 2002;17:1-16.

80. Figueiras M, Machado V, Alves N. Os modelos de senso-comum das cefaleias crónicas nos casais: relação com o ajustamento marital. Análise Psicológica 2002;1:77-90.

81. Sniehotta FF, Gorski C, Araujo-Soares V. Adoption of communitybased cardiac rehabilitation programs and physical activity following phase III cardiac rehabilitation in Scotland: a prospective and predictive study. Psychol Health 2010;25:839-54.

82. Gilbert MS. Prophylaxis: musculoskeletal evaluation. Semin Hematol 1993;30(3 Suppl 2):3-6.

83. Pettersson H, Ahlberg A, Nilsson IM. A radiologic classification of hemophilic arthropathy. Clin Orthop Relat Res 1980;149:153-9.

84. Arbuckle JL. IBM SPSS AMOS 22 users' Guide: ibm Corp. 2013. 